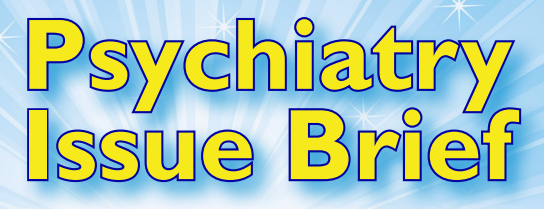

Implementation Science \& Practice

Vol 15 Iss 13

Dec 2018
A Publication of the Implementation Science \& Practice Advances Research Center A Massachusetts Department of Mental Health Research Center of Excellence

\title{
Evaluation and Impact of Trauma Informed Training on Child Professionals: UMMS Child Trauma Training Center (CTTC)
}

Zlatina Kostova, Ph.D., Jessica Griffin, Psy.D. and Genevieve Kane-Howse, LMHC

\section{Introduction}

Childhood trauma is well established as a major public health issue: statistics show that each year in the United States, upwards of one million youth experience violence, trauma and maltreatment and that by age 16 more than $67.8 \%$ of children witness or are victims of some type of violence. ${ }^{1,2}$ The cumulative impact of childhood trauma has been linked to a plethora of negative mental health and functional outcomes during adolescence and young adulthood ${ }^{3-5}$, including higher rates of re-victimization $^{6}$, aggression and delinquency.

Almost half of children with a history of trauma do not receive any services because of the wait time and lack of properly trained providers. ${ }^{8}$ More education and training is needed among child professionals to identify and respond to child trauma as soon as possible ${ }^{9}$.

\section{Who We Are}

The Child Trauma Training Center (CTTC) is a statewide program SAMHSA-funded project within the Department of Psychiatry, UMass Medical School whose mission is to improve the standard of care for traumatized youth across the Commonwealth of Massachusetts. The CTTC is bridging the above mentioned treatment gap by training child-serving professionals and systems in the state of Massachusetts and beyond, with the goal to improve trauma identification, access to trauma-informed services, and evidence-based trauma treatment. The CTTC leads a focused, multi-tiered statewide approach focusing on disseminating trauma-focused Evidence Based Practices (EBP), intensive training across service systems for our priority populations, and linking youth across the state to quality care. The CTTC has experience developing and maintaining partnerships and systemic initiatives as well as securing grant funding with the mission to:

1. Improve the identification of trauma and traumatic stress symptoms in children;

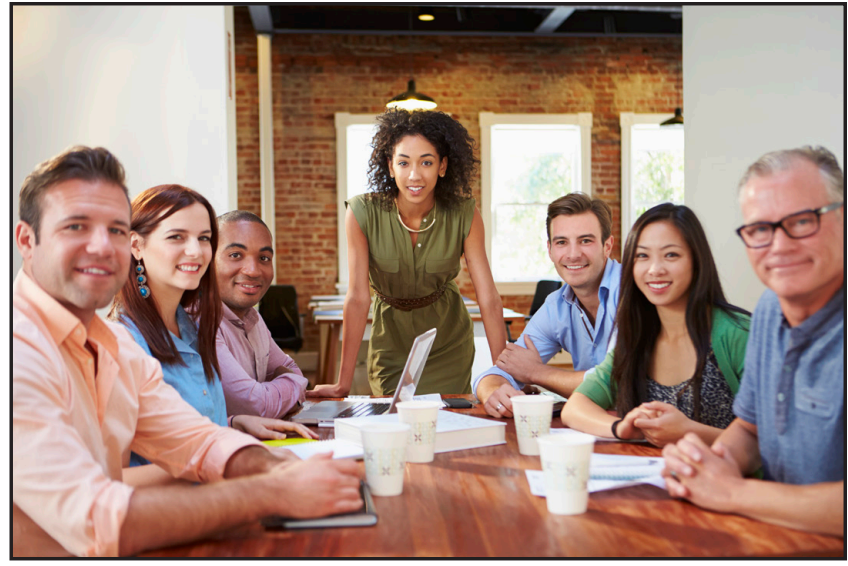

2. Improve access to treatment for youth and families who have experienced trauma and adversity; and

3. Provide clinicians who work with trauma-affected youth with training in evidence-based treatment (specifically Trauma-Focused Cognitive-Behavioral Therapy).

In this brief, we report on the program implemented by CTTC, showing how the training of professionals is a swift and efficient way to impact large numbers of children with trauma histories and ultimately to improve their lives, health, and well-being.

\section{Our Trainings}

We provide two main types of training:

- Trauma Informed Care (TIC) training for front-line, child serving professionals

- Trauma-Focused Cognitive-Behavioral Therapy (TF-CBT) for mental health clinicians

\section{Trauma Informed Care Trainings}

We disseminate Trauma Informed Care (TIC) trainings to a variety of systems, including the Department of Youth Services, Department of Children and Families, law enforcement, Probation Department, attorney groups, 
schools, as well as to parents and caregivers, nurses, medical students, and physicians. The content of this training is based on trauma training curricula developed by the National Child Traumatic Stress Network (Resource Parent Curriculum, Child Welfare Toolkit and Think Trauma Toolkit). These curricula cover a variety of topics including the consequences of trauma, impact on brain development, associated diagnosis, trauma symptoms and triggers and how professionals can intervene in a trauma-informed way. Beyond content, however, the main goal of these trainings is to change the way that professionals envision these youth and to shift their perspective from "What is wrong with this child?" to "What happened to this child?" as well as to encourage providers to be not just trauma-informed, but trauma responsive.

\section{Trauma-Focused Cognitive-Behavioral Therapy Trainings}

Trauma-Focused Cognitive-Behavioral Therapy (TF-CBT) is considered the "gold-standard" treatment in childhood trauma. It is the most rigorously tested evidence-based practice in the field, with more than twenty controlled clinical trials supporting its use across geographic, ethnic, religious and socioeconomic groups. ${ }^{10}$ In the CTTC dissemination, our training program consists of a 2-day basic training session and a 2-day advanced training session, together with monthly consultation calls for 12 months. The training curricula provide an overview of the main components of TF-CBT (PRACTICE: parenting skills, relaxation skills, affective expression, cognitive copi ng, trauma narration, in vivo mastery, conjoint child-parent sessions, enhancing future safety) as well as information about how to adapt the model to special populations such as youth with complex trauma, transition age youth, and commercially sexually exploited children. TF-CBT trainings are provided to mental health clinicians such as social workers, mental health counselors, psychologists, psychiatrists, and professionals in training.

\section{Evaluation Techniques}

We used post-evaluation surveys to assess training effectiveness in increasing participants' knowledge and skills related to child trauma. These post-evaluation surveys included objective measures of knowledge. Within the Worcester Police Department, we were able to use a more comprehensive pre- and post-training survey design.

\section{Impact}

In total, from 2012 to 2016, the Child Trauma Training Center (CTTC) provided 237 trainings. It is estimated that approximately $\mathbf{1 7 2 , 3 1 8}$ youth were impacted by the pro- fessionals who were trained, which is $\mathbf{8 6 2} \%$ of our total target goal of impacting $\mathbf{2 0 , 0 0 0}$ youth within the grant period.

\section{Trauma Informed Care Trainings}

\section{Worcester Police Department}

We provided 20 TIC trainings to 362 professionals within the Worcester Police Department, the majority of whom $(69 \%)$ were police officers. This population of trainees represent a specific and, in some ways, 'hard-to-reach' population. This training included more comprehensive pre- and post-evaluation surveys than prior trainings. The pre-test asked about the level of knowledge each participant had on prior child trauma training, prior witnessing trauma training, effect of trauma on a child's brain, effect of trauma on child development/behavior, effect of trauma reminders on behavior, effect of traumatic stress reactions on behavior, and different types of trauma exposure. These participants went into the training with low levels of knowledge of the relevant topics, rating their knowledge on average at 2.22 - well below the midpoint of 2.5 on a scale from 1 ('None') to 4 ('A lot').

Before and after the training, participants were given a six-item questionnaire testing their knowledge of various aspects of working with children who have experienced trauma. There was a statistically significant improvement $(\mathrm{p}<0.001)$ in overall questionnaire scores which, as shown by Figure 1, was driven by significant improvements on four of the six questions. While there remained gaps in their knowledge, participants clearly left the training with a better understanding of how to work with traumatized youth.

Additional feedback from police professionals indicated that the training had also boosted their subjective awareness and had given them greater insight into the young people with whom they periodically encounter in their work. They felt more confident in assessing a situation in which a child had experienced trauma, and in addressing trauma-induced challenging behavior, reflecting the two main purposes of our training.

\section{Trauma-Focused Cognitive-Behavioral Therapy Trainings}

\section{Clinicians}

Between 2012-2016, we trained 211 clinicians, mainly mental health practitioners, who work with a total of 830 clients. Since these were predominantly mental health practitioners, it is not surprising that their self-rated prior knowledge - on a similar scale from 1 ('minimal') to 4 ('great deal') - was higher than that of Worcester Police Officers. However, the average self-reported rating 
of 2.87 still indicates gaps in their knowledge about child trauma and hence room for improvement via training. The post-training survey included questions on the extent to which the training met the outlined learning objectives and increased clinicians' understanding of trauma. Average levels of agreement with a range of statements on the post-training survey indicated that participants agreed that the training was relevant to their work and practice, met their expectations, and their level of training expertise. Moreover, participants indicated a good comprehension of all the different components of TF-CBT (Figure 2).

\section{What We Learned}

» Providing training to front-line professionals and clinicians is an efficient way to reach large numbers of child victims of trauma and their families. Between 2012 and 2016, the Child Trauma Training Center (CTTC) trained 14,100 professionals who work with approximately 170,000 youth.

» Trauma-informed care training is feasible and beneficial, even with what are traditionally thought of as harder-to-reach audiences such as police officers.

» All groups of trainees had limited prior knowledge of child trauma, confirming the need to disseminate more information and training.

» Trauma-informed care trainees demonstrated a significant increase in both their (objective) knowledge and (subjective) confidence in this area.

» Clinicians believed that Trauma-Focused CognitiveBehavioral Therapy (TF-CBT) training offered many potential benefits for their work, improving their knowledge, meeting their needs, and changing their professional practice.

» By improving trauma awareness among frontline professionals, policymakers can relieve the pressure on their services and their budgets caused by delayed or erroneous identification, diagnosis and response.

\section{Conclusions}

Childhood trauma is one of the leading causes of mental health conditions and poor health outcomes. Timely diagnosis and treatment can therefore have a sizable impact on emotional and physical health. Yet, notwithstanding various state-wide efforts to improve the general standard of child welfare and mental health provision, achieving that timely diagnosis and treatment has proven difficult. One reason has been the limited awareness among childcare professionals and clinicians of trauma-informed care and evidence-based treatments. Providing training to these professionals is an effective means of remedying this problem. Evidence from the CTTC's extensive training program shows that it boosts professionals' awareness of trauma, their ability to identify it, and their understanding of how to respond in a trauma sensitive way to this vulnerable population.

This approach is effective not only with clinicians but with a range of professional groups, some of whom have not traditionally been the targets of mental health training. Our evidence shows that police officers, for example, both saw the need for the training that the CTTC provided and benefited from it. Since it is these frontline professionals who are often confronted with child victims of trauma at their most vulnerable times, it is doubly important that they too are trained to identify and to understand trauma and its effects as well as how to respond in a trauma sensitive way. Focusing training only on clinicians already quite well-versed in this area is misguided; greater efficiency lies in simultaneously coordinated outreach effort to train those professional groups who interact the most with traumatized youth on the front lines in identifying and responding to childhood trauma.

RESEARCH TEAM: Jessica L. Griffin, PsyD (Principal Investigator); Heather Forkey, MD (Co-Investigator); Genevieve Kane-Howse, LMHC (Project Director); Jessica Dym Bartlett, MSW, PhD (Evaluation Principal Investigator/Lead), Kristina Rosinsky, MPP, (Evaluation Project Director), MaryBeth Todd, MSW, Senior Research Assistant, Kerry Hynes, M.Ed., Zlatina Kostova, Ph.D., Elizabeth Lawson, BA, Diana Rinker, MA (Clinical Referral Coordinators); Laurel Post (Administrative Assistant) FUNDER: SAMHSA Grant\#S61110000033416; TIME FRAME: 2016-2021; CONTACT PERSON: Jessica.griffin@umassmed.edu

The Child Trauma Training Center, a statewide program within the Department of Psychiatry, UMass Medical School funded by a 5-year Substance Abuse Mental Health Services Administration (SAMHSA), National Child Traumatic Stress Network (NCTSN) from 2016-2021, funding from the Department of Mental Health and the Lookout Foundation. For more information: http://www.umassmed.edu/CTTC/

This is a product of Psychiatry Information in Brief. An electronic copy of this issue with full references can be found at https://escholarship.umassmed.edu/pib/vol15/iss3/1/ 
Figure 1. Differences in pre- and post-training knowledge

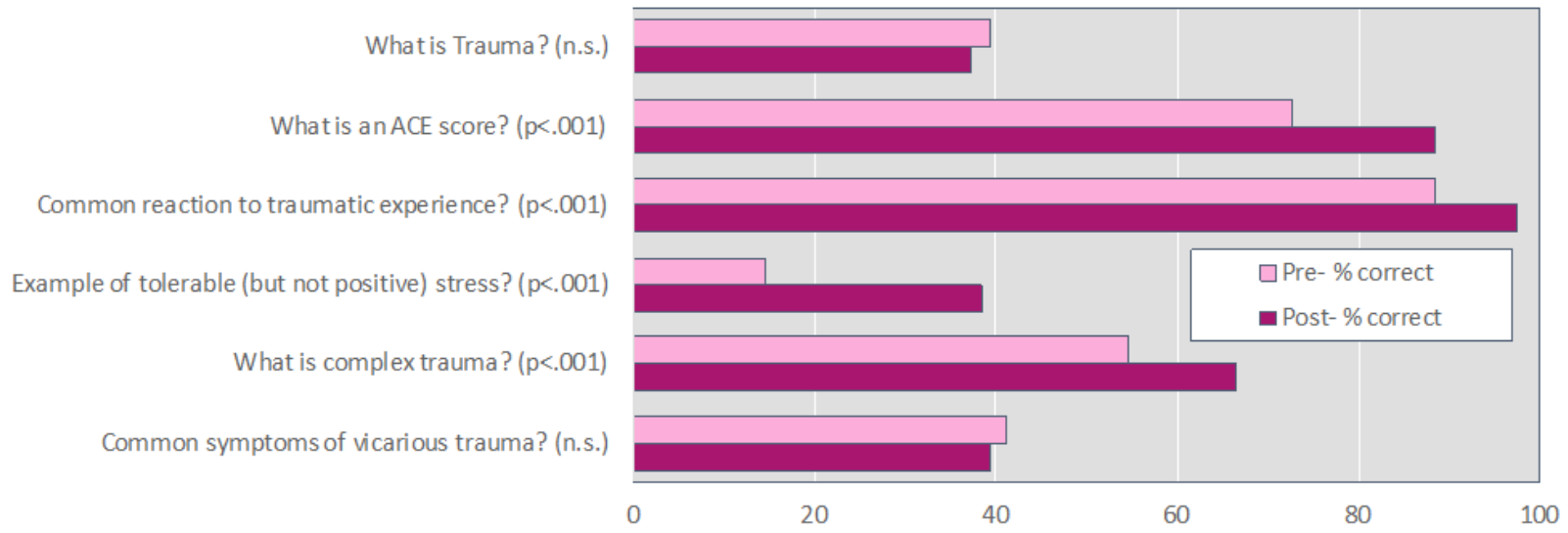

Figure 2. Learning objectives and training

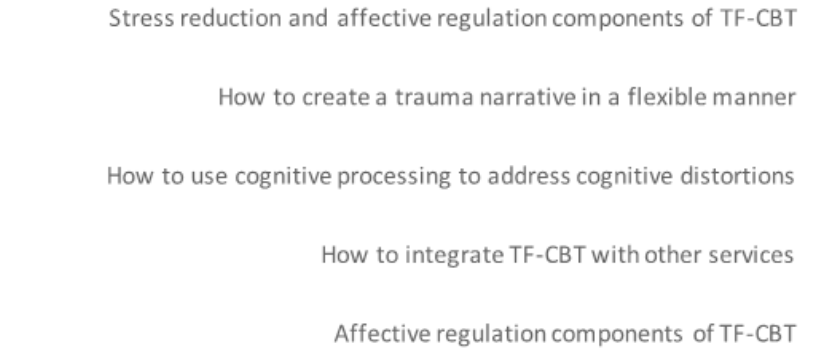

Implementing TF-CBT with those who have experienced complex trauma

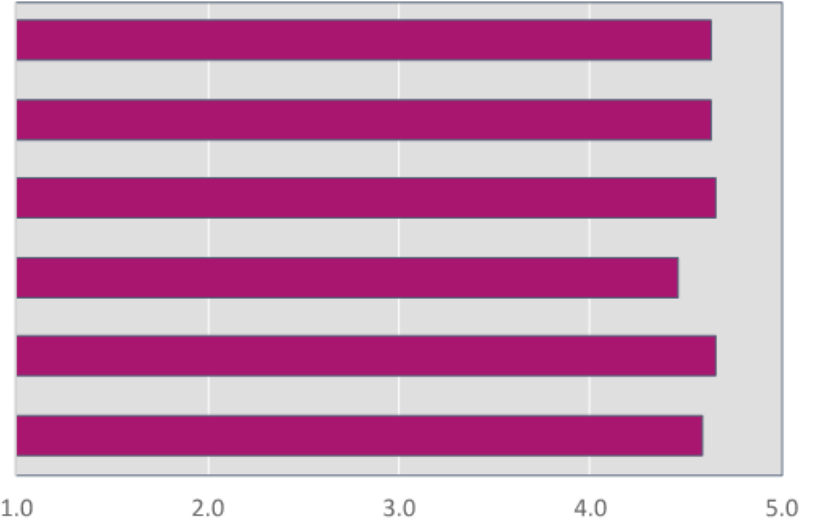

Mean agreement (1-5): "I have increased understanding of..."

${ }^{7}$ Finkelhor, D., Turner, H., Hamby, S. L., \& Ormrod, R. (2011). Polyvictimization: Children's exposure to multiple types of violence, crime, and abuse. OJJDP National Survey of Children's Exposure to Violence. Retrieved from https://www.ncjrs.gov/pdffiles1/ojidp/232273.pdf

${ }^{8}$ Cross, T. P., Jones, L. M., Walsh, W. A., Simone, M., Kolko, D., Sczepanski, J., ... \& Shadoin, A. L. (2008). Evaluating children's advocacy centers' response to child sexual abuse. OJJDP Juvenile Justice Bulletin. Retrieved from https://www.ncjrs.gov/pdffiles1/ojjdp/218530.pdf

${ }^{9}$ Shaw, M., \& De Jong, M. (2012). Child abuse and neglect: A major public health issue and the role of child and adolescent mental health services. The Psychiatrist, 36(9), 321-325. doi:10.1192/pb.bp.111.037135

${ }^{10}$ Cohen, J. A., \& Mannarino, A. P. (2015). Trauma-focused Cognitive Behavior Therapy for traumatized children and families. Child and Adolescent Psychiatric Clinics of North America, 24(3), 557-70.
${ }^{5}$ Van der Kolk, B. A., Roth, S., Pelcovitz, D., Sunday, S., \& Spinazzola, J. (2005). Disorders of extreme stress: The empirical foundation of a complex adaptation to trauma. Journal of traumatic stress, 18(5), 389-399.

${ }^{6}$ Ford, J. D., Elhai, J. D., Connor, D. F., \& Frueh, B. C. (2010). Poly-victimization and risk of posttraumatic, depressive, and substance use disorders and involvement in delinquency in a national sample of adolescents. Journal of Adolescent Health, 46(6), 545-552. 